\title{
A Intervenção Odontológica e a Visão Da Equipe Multidisciplinar em Pacientes Portadores de Parkinson do Hospital Dia Geriátrico de Anápolis
}

Beatryz Borges Magalhães Ferreira1, Bruno Gonçalves Andrade1, Sheila Cristina Assen da Costa Sales', Taynara Elias Rodrigues ${ }^{1}$ e Júlia Maria Rodrigues de Oliveira².

${ }^{1}$ Acadêmico de graduação do Curso de Odontologia do Centro Universitário de Anápolis. ${ }^{2}$ Mestre em Saúde Coletiva. Professora Adjunta do Curso de Odontologia do Centro Universitário de Anápolis.

\begin{abstract}
Resumo
Objetivo: O presente estudo tem por objetivo descrever o atendimento do Cirurgião Dentista aos pacientes com Parkinson atendidos em um Hospital Dia Geriátrico no município de Anápolis, bem como a visão da equipe multidisciplinar sobre a atuação do mesmo. Metodologia: Trata-se de um estudo descritivo transversal. A pesquisa foi realizada no Hospital Dia do Idoso de Anápolis - GO (HDI), onde os profissionais do HDI responderam ao questionário sobre o acesso aos atendimentos odontológicos realizados com os Parkinsonianos cadastrados no HDI entre o período de agosto a dezembro de 2016. Resultados: A partir da coleta de dados foi observado que $94,9 \%$ dos entrevistados da equipe multidisciplinar acharam importante indicar o paciente com Doença de Parkinson (DP) para atendimento odontológico e 53,5\% reconheceram a importância da atuação do Cirurgião-Dentista, sendo que 56,4\% dos profissionais da equipe encaminhavam os pacientes com Parkinson para atendimento odontológico. Dentre as condutas para tratamento odontológico encontradas, observou-se uma boa relação entre os cirurgiões-dentistas e os pacientes, notou-se um grande enfoque no acompanhamento preventivo, assim como o envolvimento dos cuidadores nesse processo. Conclusão: Após a análise dos resultados obtidos, verificou-se a essencialidade de um programa multidisciplinar, envolvendo todos os profissionais da saúde, em conjunto com o Cirurgião-Dentista para uma melhora da saúde bucal, física e mental, articulados em uma tarefa integrada, trazendo ao portador da doença de Parkinson uma melhor qualidade de vida.
\end{abstract}

Palavras-chave: Parkinson; Tratamento odontológico; Idoso.

\section{Introdução}

O Parkinson é uma síndrome crônica e progressiva relacionada ao sistema nervoso, apresenta sintomas como: rigidez, acinesia, bradicinesia e tremores. Sua etiologia é idiopática. Todavia, acredita-se que a Doença de Parkinson (DP), decorre de um conjunto de diversos fatores genéticos e ambientais ${ }^{1}$. O Brasil está vivenciando um crescimento da população idosa de forma rápida, devido ao aumento da expectativa de vida, o que vem gerando uma preocupação maior nos indivíduos em relação aos cuidados com a saúde. O Parkinson está na maioria das vezes associado ao processo de envelhecimento, interferindo na qualidade de vida dos portadores da doença².

Durante os procedimentos odontológicos, encontra-se uma grande dificuldade no atendimento aos pacientes Parkinsonianos devido aos sintomas cardinais da doença em conjunto com manifestações orais como, por exemplo, a rigidez muscular e sialorréia constante. Considerando o indivíduo na sua totalidade, a odontologia em conjunto com uma equipe interdisciplinar de saúde, deve garantir às futuras gerações de idosos, uma melhor saúde bucal ${ }^{3}$.

A motivação para esse estudo surgiu mediante as atuais políticas, programas, ações e legislações relacionadas à atenção com a saúde do idoso num Hospital Dia Geriátrico (HDG), não preconizarem o Cirurgião Dentista na equipe básica. Todavia, o atendimento odontológico é oferecido aos idosos do HDG de Anápolis, o que é uma iniciativa inédita e importante para uma melhor qualidade de vida dos pacientes.
Dessa forma, ao delimitar o tema deste projeto de pesquisa, considerou-se a grande importância da compreensão das limitações no atendimento ao idoso parkinsoniano, haja vista que o mesmo é dificultado devido aos tremores causados pela doença de Parkinson. Este atendimento torna-se difícil devido ao tremor que pode afetar a cabeça e a língua, intensificando a presença de sialorréia, fratura dental e trauma dos tecidos moles.

Diante do exposto, este estudo tem enquanto relevância acadêmica e social, o intuito de esclarecer a importância de um atendimento multiprofissional que envolva as famílias e cuidadores para um sucesso no tratamento odontológico ${ }^{1,13}$. O objetivo deste estudo é descrever o atendimento do Cirurgião Dentista aos pacientes com Parkinson atendidos em um Hospital Dia Geriátrico no município de Anápolis, bem como a visão da equipe multidisciplinar sobre a atuação do mesmo.

\section{Material e métodos}

Trata-se de um estudo descritivo transversal realizado com seres humanos e aprovada pelo Comitê de Ética em Pesquisa (CEP) da UniEVANGÉLICA.

A pesquisa foi realizada no Hospital Dia do Idoso (HDI), que tem como objetivo de prestar um serviço em diferentes níveis de complexidade de assistência, com uma equipe de saúde multiprofissional com dinâmica interdisciplinar. Foi solicitada a autorização à Secretaria Municipal de Saúde de Anápolis para que os profissionais do HDI pudessem responder ao questionário sobre o acesso aos atendimentos odontológicos 
realizados com os Parkinsonianos cadastrados no HDI entre o período de agosto a dezembro de 2016.

Para compor a amostra foram inclusos na pesquisa os profissionais que atuavam promovendo assistência aos pacientes portadores da doença de Parkinson no Hospital Dia do Idoso. Foram aplicados 42 questionários, divididos em 23 para a equipe multidisciplinar, considerando os fisioterapeutas, psicólogos, fonoaudiólogos, enfermeiros médicos especialistas em clínica médica, cardiologia, geriatria, 16 na equipe técnica composta por técnicos de enfermagem, administrativos, funcionários para serviços gerais e estagiários e três para a equipe da área da saúde bucal. Na amostra os profissionais inclusos foram aqueles que estavam em seu período de trabalho no Hospital Dia do Idoso durante a realização da pesquisa.

No período da coleta de dados, nove profissionais se recusaram a participar da pesquisa e, apenas um profissional se encontrava de licença de maternidade, sendo assim, foram excluídos.

Todos profissionais que trabalham no HDI foram abordados individualmente pelos pesquisadores discentes, sendo esclarecidos sobre qual era a finalidade da pesquisa, que se tratava de uma participação voluntária e não teria nenhuma penalidade caso não desejassem participar, foi assegurado total confidencialidade. Os profissionais que aceitaram participar da pesquisa foram instruídos quanto ao tempo e ao preenchimento do questionário e do Termo de Consentimento Livre e Esclarecido (TCLE). Sendo assim, todos que concordaram assinaram o TCLE.

Foi feita uma estatística descritiva na força de frequência simples e percentual. Para tanto, após os dados terem sido tabulados em uma planilha do software Microsoft Excel, estes foram transferidos para o software Statistical Package for the Social Sciences, para Windows, versão 21.0, com intuito de calcular as frequências.

Um dos riscos desta pesquisa foi o possível constrangimento dos profissionais ao responder o questionário, a omissão de dados e a recusa em participar das pesquisas, ocasionando numa possível alteração nos resultados finais. Os profissionais da categoria médica recusaram em sua maioria em responder ao questionário, porém tal comportamento não alterou os resultados da pesquisa. Esta pesquisa após a sua publicação poderá apresentar os seguintes benefícios à promoção da melhoria terapêutica dos pacientes portadores da Doença de Parkinson atendidos pelo Cirurgião-Dentista; otimizando a conduta dos profissionais quanto ao manejo desses pacientes através da sua capacitação e atualização a partir dos resultados obtidos, orientando assim os serviços que assistem a esses idosos.

As entrevistas com os profissionais foram realizadas em ambiente fechado, sem a divulgação de suas identidades e esclarecidos que poderiam a qualquer momento desistir de participar da pesquisa. Cada pesquisador discente ficou responsável para coletar os dados de um respectivo profissional.

\section{Resultados e Discussão}

A partir da coleta de dados foi observada a prevalência do sexo feminino $71,8 \%$ em relação ao masculino $28,2 \%$, a equipe que respondeu ao questionário constituiu-se de $5,1 \%$ na área de enfermagem, $30,8 \%$ na reabilitação (fisioterapeutas, fonoaudiólogos, psicólogos, assistente social), 23,1\% médicos e técnicos e 17,9\% outras áreas conforme tabela 1. A maioria dos profissionais do Hospital Dia do Idoso (HDI) atendem pacientes diagnosticados com a doença de Parkinson, equivalendo a $76,9 \%$. Os resultados mostraram que 94,9\% dos entrevistados da equipe multidisciplinar acham importante indicar o paciente com Doença de Parkinson (DP) para atendimento odontológico e 53,5\% reconheceram a importância da atuação do Cirurgião-Dentista, sendo que $56,4 \%$ dos profissionais da equipe encaminham os pacientes com Parkinson para atendimento odontológico, nas ocasiões em que se observa a higiene oral precária $(15,4 \%)$, higiene oral precária e prótese mal adaptada $(7,7 \%)$, higiene oral precária, prótese mal adaptada e dores $(5,1 \%)$, necessidade de uso de prótese $(2,6 \%)$ e prótese total mal adaptada $(2,6 \%)$ de acordo com a tabela 2. Para Alves $\mathrm{Neto}^{4}$, o Cirurgião-Dentista deve ter conhecimentos de outras áreas profissional, para que ele esteja apto a solucionar eventuais intercorrências que envolvem a boca e sua influência nos demais órgãos do organismo. A importância da equipe multidisciplinar consiste na reabilitação, educação e diagnóstico precoce, ajudando os pacientes no desenvolvimento, como também os familiares, orientando - os na prevenção e promoção de saúde, possibilitando aos pacientes uma melhor qualidade de vida.

De acordo com 94,9\% dos profissionais há outros tipos de atendimentos ao paciente com Parkinson no HDI, correspondendo a $28,2 \%$ na reabilitação; $23,1 \%$ na reabilitação e grupo de Parkinson; $15,4 \%$ reabilitação e médico; 7,7\% grupos de Parkinson; 7,7\% reabilitação e psicologia; 5,1\% reabilitação, médico e equipe de enfermagem e 2,6\% médicos. Para 5,1\%, não há nenhum programa para o atendimento ao Parkinson como demonstra a tabela 3.

Tabela 1 - Características da equipe multidisciplinar do Hospital Dia do Idoso (HDI) de Anápolis - Goiás.

\begin{tabular}{|l|l|c|c|}
\hline \multirow{3}{*}{ Sexo } & Feminino & N & $\%$ \\
\hline \multirow{3}{*}{ Área de Atuação } & Masculino & 11 & 71,8 \\
\hline & Total & 39 & 28,2 \\
\hline & Médico & 9 & 100 \\
\hline & Reabilitação & 12 & 30,8 \\
\hline & Técnico de Enfermagem & 9 & 23,1 \\
\hline & Enfermeiro & 2 & 5,1 \\
\hline & Outros & 7 & 17,9 \\
\hline & Total & 39 & 100 \\
\hline
\end{tabular}


Tabela 2 - Atendimentos à pacientes com Doença de Parkinson realizados pela equipe multidisciplinar do Hospital Dia do Idoso Anápolis - Goiás.

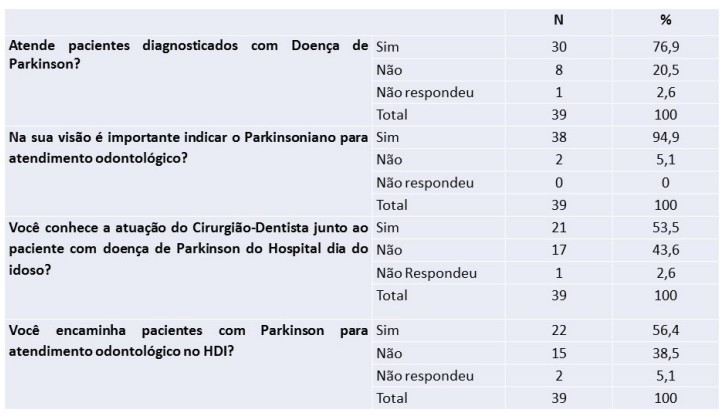

Tabela 4 - Cursos e capacitações oferecidos à equipe multidisciplinar para atuação frente aos pacientes com Doença de Parkinson.

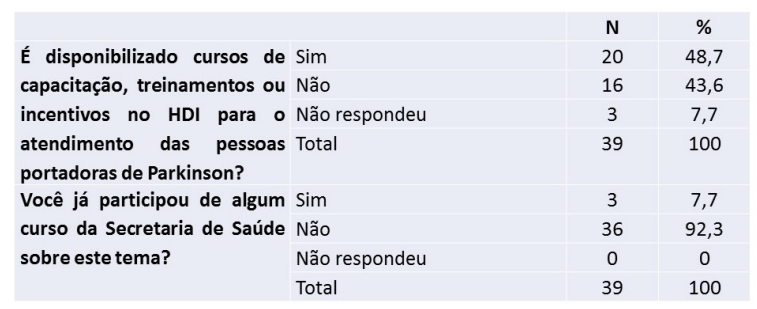

A atuação do Cirurgião-Dentista consiste em cuidados e instruções necessárias ao Parkinsoniano. Entretanto, o paciente também deverá fazer a sua parte, colaborando e aceitando o tratamento. A ação de especialistas em instituições específicas para pacientes idosos, públicas ou privadas, pode promover um completo serviço de saúde bucal para essas pessoas ${ }^{5}$.

Cerca de 43,6\% desconhecem atuação do cirurgião-dentista junto ao paciente parkisoniano no $\mathrm{HDI}$, isso ocorre devido a falta de treinamento e de uma educação permanente para equipes de saúde. Segundo El Hetti ${ }^{6}$, é fundamental, a educação continuada das equipes, pois proporciona uma releitura crítica das condições de trabalho, das relações estabelecidas e das necessidades de saúde, levando em conta as particularidades de cada região, dos usuários e dos trabalhadores envolvidos. Há uma necessidade de intensificação das ações de educação permanente para qualificar a assistência prestada, uma vez que não há domínio conceitual, nem tampouco a operacionalização dessa estratégia em vários cenários do sistema.

Por volta de $48,7 \%$ disseram que há curso de capacitação, treinamento ou incentivo no HDI para o atendimento dos parkinsonianos, porém, há uma controversa, pois $43,6 \%$ responderam que não tem esses cursos e 92,3\% nunca participaram de nenhum curso da Secretaria de Saúde sobre o tema da doença de Parkinson segundo a tabela 4. De acordo com Lemos ${ }^{7}, \mathrm{Com}$ implantação da Política Nacional de Educação Permanente em Saúde, pela Portaria n 198/GM/MS de 13 de fevereiro de 2004, a educação permanente em saúde
Tabela 3 - Relação de pacientes com Doença de Parkinson em atendimento com as diversas áreas da saúde ofertadas pelo Hospital Dia do Idoso Anápolis - Goiás.

\begin{tabular}{|c|c|c|c|}
\hline & & $\mathrm{N}$ & $\%$ \\
\hline Há pessoas com Parkinson & Sim & 38 & 94,9 \\
\hline em outros atendimentos & Não & 1 & 5,1 \\
\hline \multirow[t]{2}{*}{ além da odontologia? } & Não respondeu & 0 & 0 \\
\hline & Total & 39 & 100 \\
\hline Há pessoas com Parkinson & Reabilitação & 11 & 28,2 \\
\hline em outros atendimentos & Médicos & 1 & 2,6 \\
\hline além da odontologia? Se sim, & Grupos de Parkinson & 2 & 7,7 \\
\hline \multirow{6}{*}{ em quais programas? } & Reabilitação e Psicologia & 3 & 7,7 \\
\hline & Reabilitação e médico & 6 & 15,4 \\
\hline & $\begin{array}{l}\text { Reabilitação, médico e equipe } \\
\text { de enfermagem }\end{array}$ & 2 & 5,1 \\
\hline & $\begin{array}{l}\text { Reabilitação e Grupo de } \\
\text { Parkinson }\end{array}$ & 9 & 23,1 \\
\hline & Não respondeu & 5 & 10,2 \\
\hline & Total & 39 & 100 \\
\hline
\end{tabular}

Tabela 5 -Acessibilidade na unidade do Hospital Dia do Idoso Anápolis- Goiás.

\begin{tabular}{|c|c|c|c|}
\hline & & & \\
\hline No HDI está disponivel que & e Rampas e pisos adaptados & $\begin{array}{l}\mathrm{N} \\
4\end{array}$ & $\begin{array}{c}\% \\
10,3\end{array}$ \\
\hline meios de acessibilidade às & às Rampas e sanitários adaptados & 10 & 25,6 \\
\hline $\begin{array}{l}\text { pessoas portadoras } \\
\text { Parkinson? }\end{array}$ & $\begin{array}{l}\text { Rampas, piso, sanitários, } \\
\text { cadeira e consultório } \\
\text { adaptados }\end{array}$ & 3 & 7,7 \\
\hline & $\begin{array}{l}\text { Rampas, piso, sanitários } \\
\text { adaptados }\end{array}$ & 8 & 20,5 \\
\hline & $\begin{array}{l}\text { Rampas, sanitáriose } \\
\text { consultório adaptados }\end{array}$ & 3 & 7,7 \\
\hline & Rampas & 5 & 12,8 \\
\hline & $\begin{array}{l}\text { Rampas, piso, sanitário e } \\
\text { consultório adaptado }\end{array}$ & 1 & 2,6 \\
\hline & Piso e Sanitários adaptados & 2 & 5,1 \\
\hline & Não respondeu & 3 & 7,7 \\
\hline & Total & 39 & 100 \\
\hline
\end{tabular}

passa a ser amplamente difundida. Porém na prática os cursos tradicionais focados na proposta da educação continuada não são suficientes, pois "Capacitam-se profissionais" que "ao retornarem aos seus serviços não conseguem "aplicar" o que "aprenderam" ou constatam que o que "aprenderam" não lhes fornece elementos suficientes para enfrentar as problemáticas da realidade".

Dentre as condutas para tratamento odontológico encontradas no (HDI) observou-se uma boa relação entre os cirurgiões-dentistas e os pacientes, seguindo um protocolo com a condução cuidadosa à cadeira odontológica em posição confortável, para que o paciente possa sentir-se seguro e confiante. Presenciou-se o atendimento em posição vertical devido aos tremores e a sialorréia, dentre outros fatores que impossibilitaram a inclinação da cadeira mais que $45^{\circ}$, foram os problemas físicos apresentados pelos pacientes com Parkinson. A anamnese deve ser minuciosa para um plano de tratamento adequado. Notou-se um grande enfoque no acompanhamento preventivo sobre a higienização da prótese, as orientações devem ser centralizadas no cuidado com as mesmas.

O paciente deve tirar a prótese para a higienização e fazer a limpeza da gengiva e da língua com uma escova macia, evitando assim o surgimento de candidíase e mau hálito. É importante ressaltar a necessidade de remover a dentadura à noite. Os idosos devem ter consciência de que existe uma necessidade contínua de cuidados bucais, por isso existem meios mecânicos como a escova adaptada, que permite a 
independência do paciente ao escovar os dentes, em estágios mais avançados é necessária a ajuda de um cuidador. Deve-se estimular a autonomia do Parkinsoniano, para que ele consiga realizar suas atividades de rotina, retardando a velocidade da progressão da doença. Segundo Lins ${ }^{8}$, o atendimento deve ocorrer pela manhã, com sessões curtas, pacientes com a doença avançada o indicado é o atendimento após o uso da medicação quando estão descansados, em sessões de 60 a 90 minutos, diminuindo assim os tremores e a dificuldade de abertura de boca, facilitando o atendimento. A manutenção da higiene oral deverá ocorrer através de orientações e da utilização de produtos especializados, assim como o envolvimento dos cuidadores nesse processo.

As pessoas com a Doença de Parkinson apresentam uma deficiência motora que afeta a mobilidade e a coordenação motora geral. Em alguns casos, estes pacientes que têm problemas de locomoção conseguem andar com a ajuda de prótese, cadeira de rodas ou de outros aparelhos auxiliares ${ }^{9}$.

É de fundamental importância promover meIhorias na organização dos serviços de saúde, voltados para a garantia da acessibilidade. O HDI apresenta estruturas adequadas para a acessibilidade às pessoas com Parkinson, tendo disponível: rampas, pisos e sanitários adaptados, possibilitando a locomoção dos pacientes, principalmente àqueles em que a doença está em estado avançado que estão impossibilitados de andar e são cadeirantes, ou àqueles que andam com dificuldades e fazem uso de andadores consoante tabela 5 .

A cadeira odontológica ainda não é adaptada, o ambiente do consultório é pequeno, o que impossibilita a criação de um espaço adequado para a locomoção do paciente. Porém, o CD tem as orientações necessárias para seguir o protocolo de atendimento ao paciente com DP, diminuindo assim a dificuldade na hora do atendimento.

\section{Conclusão}

Após a análise dos resultados obtidos, verificou-se a essencialidade de um programa multidisciplinar, envolvendo fisioterapia, psicologia, terapia ocupacional, enfim, com todos os profissionais da saúde, em conjunto com o Cirurgião-Dentista para uma melhora da saúde bucal, física e mental, articulados em uma tarefa integrada, trazendo ao portador da doença de Parkinson uma melhor qualidade de vida.

A doença de Parkinson e outros problemas neurodegenerativos irão superar o câncer, sendo a principal causa de morte entre os idosos ${ }^{10}$.

Para um bom desenvolvimento do tratamento odontológico é importante uma boa interação entre o cirurgião- dentista e o paciente, pois a comunicação é prejudicada pela dificuldade do parkinsoniano em dialogar, entretanto, se houver incapacidade do paciente em expressar suas vontades durante a anamnese e início do tratamento, condutas não verbais de comu- nicação serão necessárias, assim como a presença de algum responsável. Para uma melhora da saúde oral, logo após o diagnóstico da DP, um cirurgião-dentista deve ser consultado para desenvolver um tratamento adequado, visando pouca manutenção na fase inicial e um rigoroso plano preventivo, com retornos frequentes. Um plano de tratamento adequado deve preconizar o atendimento pela manhã, com sessões curtas e em casos de pacientes com a doença avançada os procedimentos devem ser realizados num período de 60 a 90 minutos, quando o medicamento antiparkinsoniano estiver no pico de sua ação no organismo ${ }^{11}$.

Não é recomendada a inclinação da cadeira odontológica em mais de $45^{\circ}$ pelo fato deste posicionamento prejudicar a postura do cirurgião- dentista durante o atendimento, além de ser indispensável um cuidado maior, visto que, distúrbios da deglutição, poderiam levar os pacientes como DP a desenvolver pneumonia por aspiração de saliva. Odontologia preventiva é imprescindível no tratamento dentário nos pacientes com DP. A conduta inicial mais atraumática e menos invasiva possível, e a exodontia só deve ser uma opção quando nenhuma outra técnica conservadora deu resultado. O procedimento correto para o tratamento periodontal não é o cirúrgico, devido à dificuldade do controle da placa no pré e pós-cirúrgico. Uma boa opção é o tratamento não cirúrgico, que inclui: profilaxia, raspagem e alisamento coronorradicular em um pequeno intervalo de tempo. Um dos procedimentos mais efetivos feitos em pacientes com algum problema motor é a remoção de placa. Para prevenção das gengivites e outras patologias periodontais recomenda-se gluconato de clorexidina e para pacientes que não tem habilidade para cuspir é indicado um frasco com pulverizador com a solução terapêutica que é aplicado delicadamente nos tecidos orais pelo paciente ou cuidador ${ }^{12,13}$.

O atendimento multidisciplinar com profissionais de diversas áreas, como: Nutricionista, Médicos, Cirurgiões Dentistas, Auxiliares de Saúde Bucal, Equipe Administrativa, Equipe de Reabilitação (Fonoaudiologia e Fisioterapia), Equipe de Enfermagem (Enfermeiros e técnicos de enfermagem) e Equipe de Geriatria, é essencial para o sucesso do tratamento odontológico do parkinsoniano ${ }^{14}$.

\section{Referências}

1.Souza CFM, Almeida, HCP, Sousa JB, Costa PH, Silveira YSS, Bezerra JCLA. Doença de Parkinson e o Processo de Envelhecimento Motor: Uma Revisão de Literatura. Rev Neuro Mos. 2011; 19(4):718-23.

2. Navarro-peternella FM, Marcon SS. A Convivência Com a Doença de Parkinson na Perspectiva do Parkinsoniano e Seus Familiares. Rev Gau Enfer. 2010; 31(3):415-22.

3. Bulgarelli AF, Pinto LC, Rodrigues Júnior Al, Manço ARX. Estudo das queixas sobre saúde bucal em uma população de 
idosos na cidade de Ribeirão Preto-SP. Rev Bras Geria Geront. 2009; 12(2):175-91.

4. Alves Neto JSE, Morelli CC, Saranholi WA. Odontologia Na Busca De Uma Equipe Multidisciplinar Para Melhor Atendimento Às Pessoas Com Necessidades Educacionais Especiais. Ass Atend Educa Especi, 2009.

5. Rosa LB, Zuccolotto MC, Bataglion C, Coronatto EAS. Odontogeriatria - a saúde bucal na terceira. Revi Facul Odontol. 2008; 13(2):82-6.

6. El Hetti LB, Bernardes A, Gabriel CS, Fortuna CM, Mazieiro VG. Educação permanente/continuada como estratégias de gestão no Serviço de Atendimento Móvel de Urgência. Rev Eletro Enferm. 2013; 15(4):973-82.

7. Lemos CLS. Educação Permanente em Saúde no Brasil: educação ou gerenciamento permanente?. Ver Cien Sau Cole. 2016; 21(3):913-22.

8. Lins CCSA, Melo ACMA, Lima GA. Atenção à saúde bucal de idosos com Parkinson na Universidade Federal de Pernambuco: Relato de Experiência. Rev Port Divulg. 2015; 44.

9. Silva O, Panhoca L, Blanchman I. Os pacientes portadores de necessidades especiais: Revisando os conceitos de incapacidade, deficiência e desvantagem. Rev Salus. 2004, 23(1):109-16.

10. Brunetti-Montenegro FL, Marchini L. Odontogeriatria: Uma Visão Gerontológica. Rio de Janeiro: Elservier; 2013. p. 179-90.

11. Mancopes R, Busanello-Stella AR, Finger LS, Neu AP, Pacheco $A B$, Torriani MS. Influência Da Levodopa Sobre A Fase Oral Da Deglutição Em Pacientes Com Doença De Parkinson. Rev CEFAC. 2012.

12. Fiske J, Hyland K. Doença Bucal e Cuidados de Parkinson In: Atualização Dental, 2012. p. 58.

13.Campostrini E. Odontogeriatria. São Paulo: Revinter; 2004. p. 224-51.

14. Moreira CS, Martins KFC, Neri VC, Araújo PG. Doença De Parkinson: Como Diagnosticar e Tratar. Rev Cient Facul Med Camp. 2007; 2(2).
Recebido em : 23/10/2017

Aprovado em: 24/11/2017

Os autores declaram que não há conflitos de interesse.

\section{Autor correspondente:}

Julia Maria Rodrigues de Oiveira Av. Universitária Km. 3,5, Cidade Universitária, Anápolis GO, 75083-515. Contato: (62) 3310-6600.

Email: oliveira.julia@hotmail.com 


\title{
The dental intervention and the vision of the multidiscipliary team in patients with parkinson's of the geriatric hospital of Anápolis
}

\begin{abstract}
Objective: The present study aims to describe the attendance of the dentist surgeon to patients with Parkinson's attended in a geriatric day Hospital in the municipality of Annapolis, as well as the vision of the multidisciplinary team on the performance of the same. Methodology: This is a transversal descriptive study. The survey was conducted with humans and approved by the Ethics Committee in Research (CEP) of the unevangelical, at the Hospital day of the elderly of Anapolis - GO (HDI), so that the professionals of the HDI could respond to the questionnaire on access to the dental services performed With the Parkinsonian registered in the HDI between the period from August to December, 2016. Results: From the data collection it was observed that $94.9 \%$ of respondents from the multidisciplinary team found it important to indicate the patient with Parkinson's disease (DP) for dental care and 53.5\% recognized the importance of the dentist's performance, Since $56.4 \%$ of the team's professionals forwarded patients with Parkinson's to dental care. Among the ducts for dental treatment found, a good relationship was observed between dental surgeons and patients, a great focus on preventative accompaniment, as well as the involvement of caregivers in this process. Conclusion: After the analysis of the results obtained, it was found the essentiality of a multidisciplinary program involving physiotherapy, psychology, occupational therapy, at last, with all health professionals, together with the dentist for an improvement of the Oral, physical and mental health, articulated in an integrated task, bringing to the carrier of Parkinson's disease a better quality of life.
\end{abstract}

Keywords: Parkinson's; Dental treatment; Elderly. 